\title{
Strain-induced topological phase transition in phosphorene and in phosphorene nanoribbons
}

\author{
E. Taghizadeh Sisakht, ${ }^{1,2}$ F. Fazileh, ${ }^{2}$ M. H. Zare, ${ }^{3}$ M. Zarenia, ${ }^{1}$ and F. M. Peeters ${ }^{1}$ \\ ${ }^{1}$ Department of Physics, University of Antwerp, Groenenborgerlaan 171, B-2020 Antwerpen, Belgium \\ ${ }^{2}$ Department of Physics, Isfahan University of Technology, Isfahan 84156-83111, Iran \\ ${ }^{3}$ Department of Physics, Faculty of Science, Qom University of Technology, Qom 37181-46645, Iran \\ (Received 17 June 2016; revised manuscript received 2 August 2016; published 18 August 2016)
}

\begin{abstract}
Using the tight-binding (TB) approximation with inclusion of the spin-orbit interaction, we predict a topological phase transition in the electronic band structure of phosphorene in the presence of axial strains. We derive a low-energy TB Hamiltonian that includes the spin-orbit interaction for bulk phosphorene. Applying a compressive biaxial in-plane strain and perpendicular tensile strain in ranges where the structure is still stable leads to a topological phase transition. We also examine the influence of strain on zigzag phosphorene nanoribbons (zPNRs) and the formation of the corresponding protected edge states when the system is in the topological phase. For zPNRs up to a width of $100 \mathrm{~nm}$ the energy gap is at least three orders of magnitude larger than the thermal energy at room temperature.
\end{abstract}

DOI: 10.1103/PhysRevB.94.085417

\section{INTRODUCTION}

Topological insulators (TIs) with time-reversal symmetry (TRS) have been of increasing interest in condensed matter physics and materials science during the last decade. The emergence of robust edge states in two-dimensional (2D) TIs that are protected by TRS make them promising candidates for potential applications in spintronics and quantum computing [1-6]. TIs can exist intrinsically or be driven by external factors such as electrical field or by functionalization [7]. Strain engineering is a well-known strategy for switching from the normal-insulator (NI) phase to a TI phase [7,8]. Among the wide list of systems that possess such property, 2D materials with fascinating electronic, mechanical, and thermal properties have been in the focus of attention [1,9].

In the past few years, phosphorene, a monolayer of black phosphorus, has emerged as an encouraging 2D semiconducting material for widespread applications. Phosphorene-based field effect transistors (FETs) show a higher ON/OFF ratio in comparison with graphene [10,11] and have a higher carrier mobility with respect to 2D transition-metal dichalcogenides (TMDs), which have recently attracted a lot of attention for FET applications [10-12]. There exist several works pertinent to the observation of different phases in bulk and multilayer black phosphorous by tuning the lowest energy bands [13-17]. Using density functional theory (DFT) it was shown that few-layer phosphorene experiences a NI to TI and then a TI to topological metal (TM) phase transition by applying a perpendicular electric field [16]. In a different DFT study [17] such phase transitions for various stacked bilayer phosphorene under in-plane strain have been explored. Owing to the puckered structure of phosphorene, it has a high degree of flexibility. Therefore, it can sustain strain very well specially in the zigzag direction up to about $30 \%$ [18,19]. This makes phosphorene promising for possible applications using strain engineering.

In our work, we investigate the effect of strain on the electronic band structure of phosphorene within the TB approach. The band gaps of this model [20] are close to the most reliable DFT and experimental results [21,22] that predict band gaps of $1 \sim 2 \mathrm{eV}$ for phosphorene. In this paper, we propose a model Hamiltonian for the spin-orbit coupling (SOC) for monolayer phosphorene that can be generalized to few-layer phosphorene. We show that a model which includes the next-nearest (n-n) neighbors in the upper or lower chains is sufficient for capturing the main physics. Then, strain engineering of this system is investigated through modifying the hopping parameters of the system. We demonstrate that, by applying particular types of strain, the system can make a phase transition to a TI. Finally, we show numerically that although the topological bulk band gap induced by SOC is about $5 \mathrm{meV}$, the highly anisotropic nature of this material causes the corresponding bulk gaps in large-width zigzag phosphorene nanoribbons (zPNRs) to be at least three orders of magnitude larger than room-temperature thermal energy ( $\sim 26 \mathrm{meV}$ ) and makes phosphorene nanoribbons excellent candidates for future applications.

This paper is organized as follows: The effective low-energy TB model Hamiltonian including the SOC terms is obtained in Sec. II. The effect of axial strains on the band structure produced by this model is calculated and our results are compared with DFT results in Sec. III. Demonstration of a topological phase transition in the electronic properties of phosphorene when particular types of strain are applied and the characteristics of corresponding edge states in zPNRs are presented in Sec. IV. The paper is summarized in Sec. V.

\section{TIGHT-BINDING MODEL HAMILTONIAN INCLUDING SPIN-ORBIT INTERACTION}

\section{A. Structure}

The puckered atomic structure of phosphorene and its geometrical parameters are shown in Fig. 1, where the $x$ and $y$ axes are the armchair and zigzag directions, respectively, and the $z$ axis is in the normal direction to the plane of phosphorene. With this definition of coordinates, one can indicate the various atom connections $r_{i}$ which correspond to various hopping parameters $t_{i}$ that are included in the TB model. The structure parameters have been taken from [23] which is very close to experimentally measured parameters [24] for its bulk structure. The components of the geometrical parameters as shown in Figs. 1(b) and 1(c), for bond lengths $r_{1}=$ $2.240 \AA$ and $r_{2}=2.280 \AA$, are $\left(r_{1 x}, r_{1 y}, r_{1 z}\right)=(1.503,1.660,0)$ and $\left(r_{2 x}, r_{2 y}, r_{2 z}\right)=(0.786,0,2.140)$, and $r_{3}, r_{4}, r_{5}$ are simply 
(a)

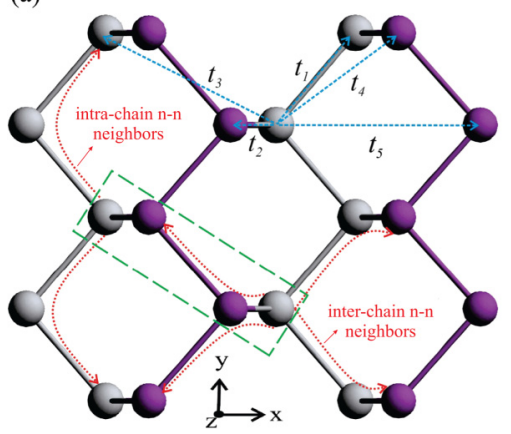

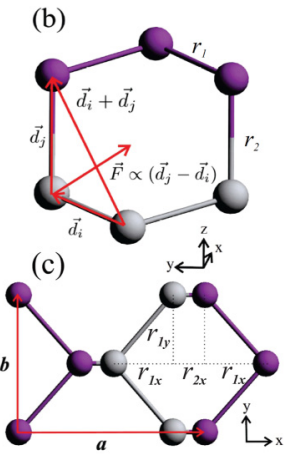

FIG. 1. The lattice geometry of phosphorene. The two different colors of the $\mathrm{P}$ atoms refer to upper and lower chains. (a) The hopping parameters $t_{1}, t_{2}, \ldots, t_{5}$ used in our TB model are indicated in the figure. Red dotted arrows represent two types of n-n neighbors and the green dashed rectangle shows the unit cell of phosphorene. (b) A honeycomb-like ring of phosphorene. The vectors $\vec{d}_{i}, \vec{d}_{j}, \vec{d}_{i}+\overrightarrow{\vec{d}}_{j}$, and $\vec{F} \propto\left(\vec{d}_{j}-\vec{d}_{i}\right)$ are used to derive the SOC. (c) Lattice constants and the components of geometrical parameters describing the structure of phosphorene.

defined by parameters of $r_{1}$ and $r_{2}$. The two in-plane lattice constants are $a=4.580 \AA, b=3.320 \AA$ and the thickness of a single layer due to the puckered nature is $r_{2 z}=2.140 \AA$.

\section{B. Tight-binding model}

The phosphorene TB Hamiltonian that has been proposed earlier [20], without the spin degree of freedom, is given by

$$
\hat{H}=\sum_{i, j} t_{i j} c_{i}^{\dagger} c_{j}
$$

where the summation is up to fifth neighbors, and $t_{i j}$ are hopping integrals that show the energy transfer between the $i$ th and $j$ th sites. The hopping terms are shown in Fig. 1(a). $c_{i}^{\dagger}$ and $c_{j}$ represent the creation and annihilation operators of electrons in sites $i$ and $j$, respectively. The numerical values of these hopping parameters are $t_{1}=-1.220 \mathrm{eV}, t_{2}=3.665 \mathrm{eV}$, $t_{3}=-0.205 \mathrm{eV}, t_{4}=-0.105 \mathrm{eV}$, and $t_{5}=-0.055 \mathrm{eV}$ [20]. Including the spin degree of freedom and SOC the Hamiltonian is modified into

$$
\hat{H}=\sum_{i, j, \alpha} t_{i j} c_{i \alpha}^{\dagger} c_{j \alpha}+\hat{H}_{S O}
$$

where in $\hat{H}_{S O}=\hat{H}_{S O 1}+\hat{H}_{S O 2}$, the first term is called the usual effective SOC and the second one is the intrinsic Rashba SOC which will be introduced in next subsection. Due to the puckered structure of phosphorene, the Rashba term is rather large as compared to the first term and should be included in our calculations.

\section{Spin-orbit coupling in phosphorene}

The primary goal of this subsection is to introduce a spin-orbit model Hamiltonian for phosphorene which can capture the most important spin-related phenomenon. There exist several studies which show the anisotropic behavior in the electronic and optical properties of phosphorene [22,25-27] which are due to the anisotropic nature of the band dispersion of phosphorene. This property is reflected in the effective

mass of electrons and holes of phosphorene. As a matter of fact, the corresponding band dispersion of the zigzag direction in real space is relatively flat near the Fermi energy while it has an approximately linear dispersion in the armchair direction [25,27]. One can define two types of $n-n$ neighbors in the phosphorene structure. As shown in Fig. 1(a), each $\mathrm{P}$ atom has two intrachain and four interchain n-n neighbors, respectively. The effective mass of electrons in the intrachain direction are at least an order of magnitude larger than in the interchain direction [25]. Therefore, electrons usually select the interchain path for circular motion, allowing us to ignore the intrachain neighbors and only consider the four $n-n$ interchain $\mathrm{P}$ atoms in the SOC model.

In general, the $\mathrm{SOC}$ term for a $2 \mathrm{D}$ system is given by

$$
H_{S O}=-\frac{\hbar}{4 m_{0}^{2} c^{2}}(\vec{F} \times \vec{P}) \cdot \vec{\sigma},
$$

where $\hbar, m_{0}$, and $c$ are Planck's constant, mass of the free electron, and the velocity of light, respectively. $\vec{F}$ is the effective electrostatic force, $\vec{P}$ is the effective momentum, and $\vec{\sigma}$ denotes the Pauli matrices. As in the cases of graphene and silicene [28], the nearest-neighbor SOC is zero in phosphorene, but the SOC terms of the n-n neighbors are nonzero.

As shown in Fig. 1(b), in a honeycomb-like ring of phosphorene, we can define $\vec{d}_{i}$ and $\vec{d}_{j}$ as vectors that connect the nearest $\mathrm{P}$ atoms to each other and $\vec{d}_{i}+\vec{d}_{j}$ the connecting vector of n-n neighbors. Using these vectors, the electrostatic force and momentum can be written as $\vec{F}=|\vec{F}|\left(\vec{d}_{j}-\vec{d}_{i}\right) /\left|\vec{d}_{j}-\vec{d}_{i}\right|$ and $\vec{P}=-i \hbar \vec{\nabla} \equiv-i \alpha\left(\vec{d}_{i}+\vec{d}_{j}\right)$, with $\alpha$ being a prefactor. Rewriting the SOC in terms of the above definitions we obtain

$$
H_{S O}=-\frac{\hbar}{4 m_{0}^{2} c^{2}}\left[\frac{|\vec{F}|(-i \alpha)}{\left|\vec{d}_{j}-\vec{d}_{i}\right|}\left(\vec{d}_{j}-\vec{d}_{i}\right) \times\left(\vec{d}_{i}+\vec{d}_{j}\right)\right] \cdot \vec{\sigma} .
$$

Based on experimental and DFT data, $\left|\vec{d}_{i}\right|$ and $\left|\vec{d}_{j}\right|$ are approximately equal $[18,23,24,29]$; therefore $\left(\vec{d}_{i}+\vec{d}_{j}\right)$ and $\left(\vec{d}_{j}-\vec{d}_{i}\right)$ become perpendicular to each other. This leads to

$H_{S O}=-i \frac{2 \hbar \alpha|\vec{F}|}{4 m_{0}^{2} c^{2}\left|\vec{d}_{j}-\vec{d}_{i}\right|}\left(\vec{d}_{i} \times \vec{d}_{j}\right) \cdot \vec{\sigma} \equiv-i \gamma\left(\vec{d}_{i} \times \vec{d}_{j}\right) \cdot \vec{\sigma}$,

where the term $2 \hbar \alpha|\vec{F}| / 4 m_{0}^{2} c^{2}\left|\vec{d}_{j}-\vec{d}_{i}\right|=\gamma$ will be adjusted to obtain the correct value of SOC as obtained by DFT. Notice that the above approximations reduce the two parameters of the usual SOC and intrinsic Rashba SOC into a single parameter. Using $\vec{\sigma}=\sigma_{\|} \hat{a}_{\|}+\sigma_{z} \hat{a}_{z}$, where $\sigma_{\|}\left(\sigma_{z}\right)$ are the in-plane (outof-plane) Pauli matrices (matrix), we rewrite Eq. (5) as

$$
H_{S O}=-i \gamma\left|\vec{d}_{i} \times \vec{d}_{j}\right|_{z} v_{i j} \sigma_{z}-i \gamma\left|\left(\vec{d}_{i} \times \vec{d}_{j}\right)_{\|}\right|\left(\vec{d}_{i} \times \vec{d}_{j}\right)_{\|}^{0} \cdot \vec{\sigma}_{\|},
$$

where $v_{i j} \equiv\left(\vec{d}_{i} \times \vec{d}_{j}\right)_{z} /\left|\vec{d}_{i} \times \vec{d}_{j}\right|_{z}= \pm 1$ and $\left(\vec{d}_{i} \times \vec{d}_{j}\right)_{\|}^{0} \equiv$ $\left(\vec{d}_{i} \times \vec{d}_{j}\right)_{\| 1} /\left|\left(\vec{d}_{i} \times \vec{d}_{j}\right)_{\|}\right|$is a dimensionless unit vector. The spin-orbit terms in second quantization are given by

$$
\begin{aligned}
\hat{H}_{S O 1}+\hat{H}_{S O 2}= & -i \lambda_{s o} \sum_{\langle i j\rangle \alpha \beta} v_{i j} c_{i \alpha}^{\dagger} \sigma_{z}^{\alpha \beta} c_{j \beta} \\
& -i \lambda_{r} \sum_{\langle i j\rangle\rangle \alpha \beta} c_{i \alpha}^{\dagger}\left(\vec{d}_{i} \times \vec{d}_{j}\right)^{0} \cdot \vec{\sigma}_{\|}^{\alpha \beta} c_{j \beta},
\end{aligned}
$$


where $\lambda_{s o} \equiv \gamma\left|\vec{d}_{i} \times \vec{d}_{j}\right|_{z}$ and $\lambda_{r} \equiv \gamma\left|\left(\vec{d}_{i} \times \vec{d}_{j}\right)_{\|}\right|$are effective intrinsic SOC and intrinsic Rashba constants, and the summation runs over the interchain n-n neighbors. As mentioned before, these two parameters are related to one parameter $\gamma$, which can be estimated by adjusting the TB band structure of phosphorene to the one obtained from DFT. It was shown that in the absence of SOC the energy gap of few-layer phosphorene closes under an external electric field or strain [16,17]. However, including the SOC an energy gap of $5 \mathrm{meV}$ [16] remains in few-layer phosphorene. This results in the value of $\gamma \approx 0.006 \mathrm{meV} / \AA^{2}$ in our TB model.

\section{PHOSPHORENE UNDER STRAIN: ELECTRONIC BAND STRUCTURE}

The role of uniaxial and biaxial strain in manipulating the electronic structure of few-layer phosphorene has been investigated via DFT [17,19,30-32] and TB approaches [33-35]. Applying tensile or compressive strain in different directions results in different modifications of the electronic bands. One can observe a direct-to-indirect gap transition, or a prior direct band gap closing, depending on the type of applied strain $[17,19,31]$. In this work we consider biaxial compressive strain in the plane of few-layer phosphorene [17,31], and tensile strain in the normal direction [32]. This modifies the low-energy bands so that the valance and conduction bands approach each other. By further increasing strain, the lower band, coming from $p_{x}$ orbitals, shifts upward resulting in a semimetal phase [31] given that at the band crossing point a minigap opens due to the SOC. Investigating the local density of states of $p$ orbitals [17] shows that our one-orbital $p_{z}$-like TB model is still valid in the low-energy limit before the semimetal phase appears.

In the following, we will first study the bulk band of phosphorene in the presence of axial strains using our TB approach and demonstrate that a band inversion occurs in the energy spectrum of phosphorene in the range where the structure is still stable under strain. It has been shown that the bond lengths and bond angles of phosphorene both change under axial strains $[31,36]$. Therefore, the hopping parameters will change. According to the Harrison rule [37,38], the hopping parameters for $p$ orbitals are related to the bond length as $t_{i} \propto 1 / r_{i}^{2}$ and the angular dependence can be described by the hopping integrals along the $\pi$ and $\sigma$ bonds. However, our calculations showed that although the changes in angles are almost noticeable $[31,36]$, the modification of the hopping parameters due to them is much smaller than the effect of changes of bond lengths. Hence, we consider only changes of the bond lengths in the hopping modulation.

When an axial strain is applied to phosphorene, the rectangle shape of the unit cell with lattice constants of $a_{0}$ and $b_{0}$ remains unchanged. Therefore the initial geometrical parameter $r_{i}^{0}$ is deformed as $\left(r_{i x}, r_{i y}, r_{i z}\right)=\left(\left(1+\varepsilon_{x}\right) r_{i x}^{0}\right.$, $\left.\left(1+\varepsilon_{y}\right) r_{i y}^{0},\left(1+\varepsilon_{z}\right) r_{i z}^{0}\right)$, where $\varepsilon_{j}$ is the strain in the $j$ direction and $r_{i}$ is a deformed geometrical parameter. In the linear deformation regime, expanding the norm of $r_{i}$ to first order of $\varepsilon_{j}$ gives

$$
r_{i}=\left(1+\alpha_{x}^{i} \varepsilon_{x}+\alpha_{y}^{i} \varepsilon_{y}+\alpha_{z}^{i} \varepsilon_{z}\right) r_{i}^{0},
$$

where $\alpha_{j}^{i}=\left(r_{i j}^{0} / r_{i}^{0}\right)^{2}$ are coefficients related to the structure of phosphorene which are simply calculated via the special geometrical parameters given in previous section. Using the Harrison relation, we obtain the strain effect on the hopping parameters as

$$
t_{i} \approx\left(1-2 \alpha_{x}^{i} \varepsilon_{x}-2 \alpha_{y}^{i} \varepsilon_{y}-2 \alpha_{z}^{i} \varepsilon_{z}\right) t_{i}^{0},
$$

where $t_{i}$ is the modified hopping parameter of deformed phosphorene with new lattice constants $a$ and $b$.

Let us now study the energy spectrum of strained phosphorene with the modified hopping parameters as given by Eq. (9). The unit cell of monolayer phosphorene is a rectangle containing four atoms as shown in Fig. 1(a). The Fourier transform of the strained Hamiltonian of Eq. (2) gives the general Hamiltonian in momentum space as

$$
H=\sum_{\mathbf{k}} \psi_{\mathbf{k}}^{\dagger} H_{\mathbf{k}} \psi_{\mathbf{k}},
$$

where we have used the basis $\psi_{\mathbf{k}}^{\dagger}=\left\{a_{\mathbf{k}}^{\dagger}, b_{\mathbf{k}}^{\dagger}, c_{\mathbf{k}}^{\dagger}, d_{\mathbf{k}}^{\dagger}\right\} \otimes\{\uparrow, \downarrow\}$ with $H_{\mathbf{k}}$ being

$$
H_{\mathbf{k}}=\left(\begin{array}{ll}
H_{\mathbf{k}}(\uparrow \uparrow) & H_{\mathbf{k}}(\uparrow \downarrow) \\
H_{\mathbf{k}}(\downarrow \uparrow) & H_{\mathbf{k}}(\downarrow \downarrow)
\end{array}\right),
$$

where

$$
\begin{aligned}
& H_{\mathbf{k}}(\uparrow \uparrow)=H_{\mathbf{k}}^{(4)}+H_{\mathbf{k}}^{\text {so }}, \quad H_{\mathbf{k}}(\downarrow \downarrow)=H_{\mathbf{k}}^{(4)}-H_{\mathbf{k}}^{(\mathrm{so})}, \\
& H_{\mathbf{k}}(\uparrow \downarrow)=H_{\mathbf{k}}^{(R)}, \quad H_{\mathbf{k}}(\downarrow \uparrow)=H_{\mathbf{k}}^{\dagger^{(R)}}
\end{aligned}
$$

are $4 \times 4$ matrices

$$
\begin{aligned}
H_{\mathbf{k}}^{(4)} & =\left(\begin{array}{cccc}
0 & A_{\mathbf{k}} & B_{\mathbf{k}} & C_{\mathbf{k}} \\
A_{\mathbf{k}}^{*} & 0 & D_{\mathbf{k}} & B_{\mathbf{k}} \\
B_{\mathbf{k}}^{*} & D_{\mathbf{k}}^{*} & 0 & A_{\mathbf{k}} \\
C_{\mathbf{k}}^{*} & B_{\mathbf{k}}^{*} & A_{\mathbf{k}}^{*} & 0
\end{array}\right), \\
H_{\mathbf{k}}^{(\mathrm{so})} & =\left(\begin{array}{cccc}
0 & 0 & E_{\mathbf{k}} & 0 \\
0 & 0 & 0 & -E_{\mathbf{k}} \\
E_{\mathbf{k}}^{*} & 0 & 0 & 0 \\
0 & -E_{\mathbf{k}}^{*} & 0 & 0
\end{array}\right), \\
H_{\mathbf{k}}^{(R)} & =\left(\begin{array}{ccccc}
0 & 0 & F_{\mathbf{k}} & 0 \\
0 & 0 & 0 & F_{\mathbf{k}} \\
e^{i\left(k_{a}-k_{b}\right)} F_{\mathbf{k}} & 0 & 0 & 0 \\
0 & e^{i\left(k_{a}-k_{b}\right)} F_{\mathbf{k}} & 0 & 0
\end{array}\right),
\end{aligned}
$$

whose elements are given by

$$
\begin{aligned}
A_{\mathbf{k}}= & t_{2}+t_{5} e^{-i k_{a}}, \\
B_{\mathbf{k}}= & 4 t_{4} e^{-i\left(k_{a}-k_{b}\right) / 2} \cos \left(k_{a} / 2\right) \cos \left(k_{b} / 2\right), \\
C_{\mathbf{k}}= & 2 e^{i k_{b} / 2} \cos \left(k_{b} / 2\right)\left(t_{1} e^{-i k_{a}}+t_{3}\right), \\
D_{\mathbf{k}}= & 2 e^{i k_{b} / 2} \cos \left(k_{b} / 2\right)\left(t_{1}+t_{3} e^{-i k_{a}}\right), \\
E_{\mathbf{K}}= & -2 \lambda_{s o} e^{-i\left(k_{a}-k_{b}\right) / 2} \sin \left(k_{a} / 2\right) \sin \left(k_{b} / 2\right), \\
F_{\mathbf{K}}= & 4 \lambda_{r} e^{\left(k_{b}-k_{a}\right) / 2}\left(\cos \left(k_{b} / 2\right) \cos \left(k_{a} / 2\right) \cos (\theta),\right. \\
& \left.+i \sin \left(k_{b}\right) \sin \left(k_{a}\right) \sin (\theta)\right),
\end{aligned}
$$

with $k_{a}=\mathbf{k} \cdot \mathbf{a}, k_{b}=\mathbf{k} \cdot \mathbf{b}$, and $\theta=\arctan \left(r_{1 y} / r_{1 x}\right)$.

The energy spectrum of pristine phosphorene in the absence of strain has been obtained by numerical diagonalization of the 

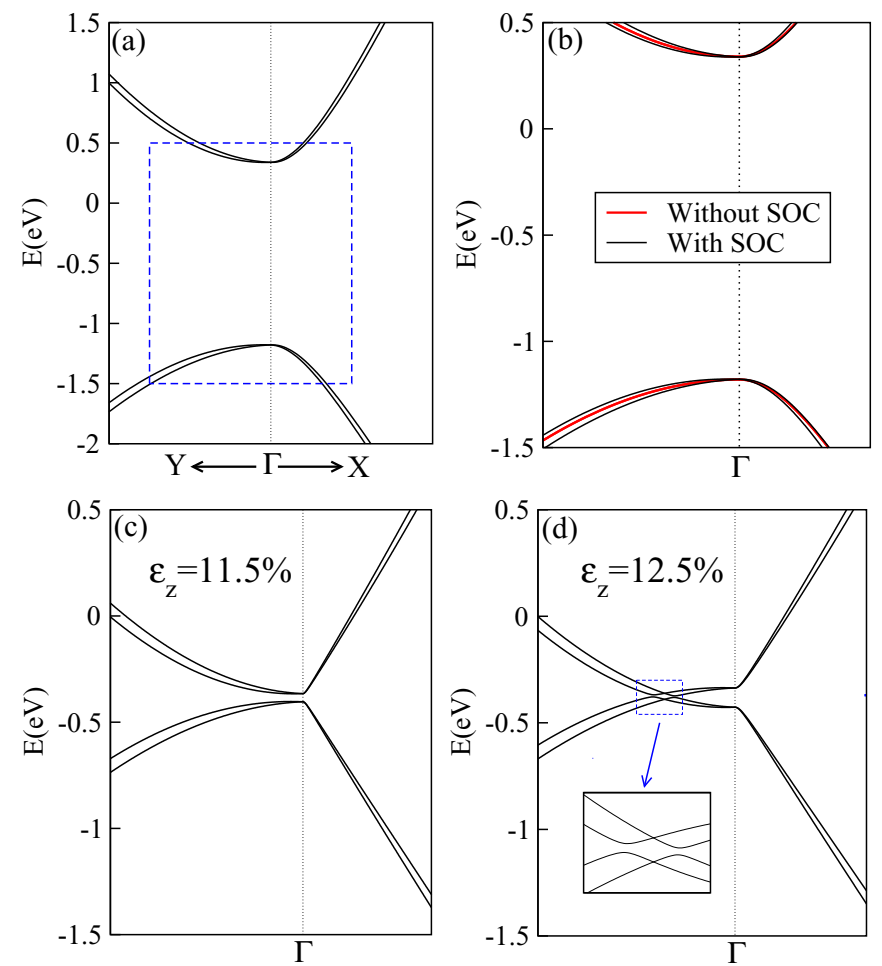

FIG. 2. (a) The TB bands of phosphorene including the effect of SOC. The blue dashed rectangle is magnified in (b), (c), and (d) for various conditions: (b) The magnified valence and conduction bands of phosphorene. Red curves show the bands without SOC. Black solid curves show the bands with SOC. (c), (d) The energy spectrum right before and after band inversion at $11.5 \%$ and $12.5 \%$ perpendicular tensile strain, respectively. The inset shows the gap opening due to the SOC, i.e., $\sim 5 \mathrm{meV}$.

TB Hamiltonian Eq. (10) in different symmetry directions as shown in Fig. 2(a). As we can see in Fig. 2(b), the degeneracies of bands have been removed (black lines) slightly due to the SOC in comparison with the case of zero SOC coupling (red lines) except for the time-reversal-invariant momenta (TRIMs) which are at least doubly degenerate according to the Kramers theorem.

As seen in Fig. 2 the gap of phosphorene is located at the $\Gamma$ point which is also a TRIM. At this point, the spin-up and spin-down valence and conduction bands are degenerate and the change in the gap due to the SOC is very small as compared to the bulk gap. Since axial strain does not break TRS, the bands at this point remain degenerate. Therefore, when the bulk gap is modified by an external factor such as strain, we can safely use the spinless Hamiltonian demonstrating the general trend in changes of the gap. All $\mathrm{P}$ atoms in a unit cell have the same on-site energy, so we can project the position of upper and lower chains of phosphorene on a horizontal plane to reduce the spinless $4 \times 4$ Hamiltonian $H_{\mathbf{k}}^{(4)}$ into a two-band TB model $[27,39]$. Therefore the new $k$-space Hamiltonian of the strained phosphorene in the absence of spin is given by

$$
H_{\mathbf{k}}^{(2)}=\left(\begin{array}{cc}
B_{\mathbf{k}} e^{i\left(k_{a}-k_{b}\right) / 2} & A_{\mathbf{k}}+C_{\mathbf{k}} e^{i\left(k_{a}-k_{b}\right) / 2} \\
A_{\mathbf{k}}^{*}+C_{\mathbf{k}}^{*} e^{-i\left(k_{a}-k_{b}\right) / 2} & B_{\mathbf{k}} e^{i\left(k_{a}-k_{b}\right) / 2}
\end{array}\right)
$$

Diagonalizing this Hamiltonian at the $\Gamma$ point gives the band gap as

$$
\begin{aligned}
E_{g}= & \left(4 t_{1}^{0}+2 t_{2}^{0}+4 t_{3}^{0}+2 t_{5}^{0}\right) \\
& -\sum_{j}\left(8 \alpha_{j}^{1} \varepsilon_{j} t_{1}^{0}+4 \alpha_{j}^{2} \varepsilon_{j} t_{2}^{0}+8 \alpha_{j}^{3} \varepsilon_{j} t_{3}^{0}+4 \alpha_{j}^{5} \varepsilon_{j} t_{5}^{0}\right),
\end{aligned}
$$

where $j$ denotes the summation over $x, y, z$ components. The first bracket is the unstrained band gap, i.e., $E_{g}^{0}=1.52 \mathrm{eV}$, and the second one indicates the structurally dependent values of change in the band gap due to the axial strains. Inserting the numerical values of the structural parameters in Eq. (16) we obtain a compact form for the gap equation

$$
E_{g}=E_{g}^{0}-\sum_{j} \eta_{j} \varepsilon_{j}
$$

where $\eta_{x}=-4.09 \mathrm{eV}, \eta_{y}=-5.72 \mathrm{eV}$, and $\eta_{z}=12.86 \mathrm{eV}$. Equation (17) shows that by applying in-plane compressive biaxial strain and perpendicular tensile strain, the band gap decreases, which is consistent with DFT calculations [17,19,30-32]. It is shown that DFT calculations using the PBE exchange correlation functional anticipate properly the general trends of the band structure when applying axial strains on phosphorene [19,31]. A comparison between the band gaps as function of axial strains using available DFT data [19,31,32] and the TB model demonstrates that the modification of the hopping parameters in the linear regime is valid for rather large strains and shows that the modified TB model predicts correctly the variation of the low-energy spectrum. Figure 3 shows the band gap values evaluated at the $\Gamma$ point in the presence of (a) uniaxial perpendicular tensile strain, (b) uniaxial compressive strain in the armchair direction, and (c) biaxial compressive in-plane strain, respectively. In both DFT and TB approaches the band gaps exhibit linear dependence with applied strain. The discrepancy between the values of the band gaps originates from the specific calculation method.

As a particular case we consider the modification of energy of the spectrum under a perpendicular tensile strain. By increasing the tensile strain, a band inversion occurs at the critical value of $\varepsilon_{z}^{c}=E_{g}^{0} / \eta_{z}=0.118$. This is a signal of a topological phase transition. Figures 2(c) and 2(d) show the low-energy bands just before and after band closing at $11.5 \%$ and $12.5 \%$ tensile strain, respectively. As shown in the inset of Fig. 2(d), the SOC opens a small gap of about $5 \mathrm{meV}$ after band closing preventing the formation of a Dirac-like cone.

Notice from Fig. 2 that the low-energy bands in the armchair direction become more linear under strain. This makes the intrachain n-n neighbors less important justifying the use of the SOC terms of Eq. (7).

\section{TOPOLOGICAL PHASE TRANSITION OF PHOSPHORENE UNDER STRAIN}

The $\mathbb{Z}_{2}$ classification provides a very strong distinction between two different time-reversal topological and trivial phases. Pristine phosphorene as a trivial insulator when the intrinsic SOC effect is included preserves the TRS and can exhibit a quantum spin Hall (QSH) phase when its electronic properties are influenced by external factors, e.g., electric field or strain. In the following, we first briefly describe 

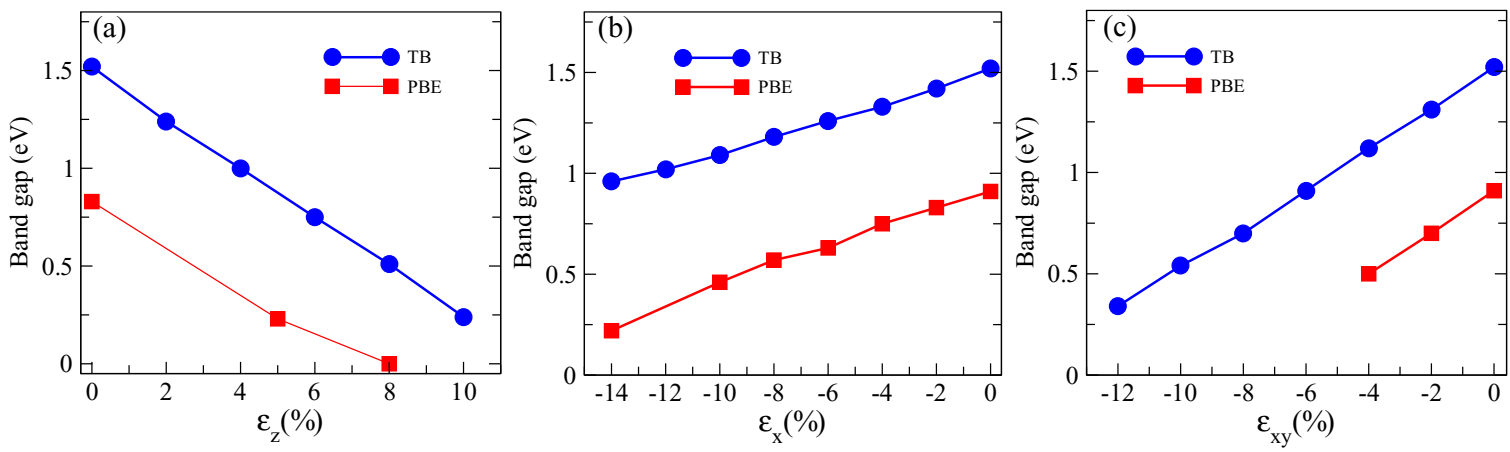

FIG. 3. Band gap evolution of phosphorene in the presence of (a) perpendicular tensile strain, (b) uniaxial compressive strain in armchair direction, and (c) biaxial compressive in-plane strain.

our approach for calculating the $\mathbb{Z}_{2}$ invariant. This approach, when working in the frame of the TB model [40], is quite efficient for 2D materials such as phosphorene. Then, we will demonstrate numerically a topological phase transition in strained phosphorene and calculate the phase diagrams accordingly. Finally we will show the existence of protected edge states in zPNRs and discuss their fascinating properties.

\section{A. Calculation of $\mathbb{Z}_{2}$ invariant}

Fu and Kane [41] showed that an equivalent way to calculate the $\mathbb{Z}_{2}$ invariant is as an integral over half the Brillouin zone given by

$\mathbb{Z}_{2}=\frac{1}{2 \pi i}\left[\oint_{\partial \mathrm{HBZ}} d \boldsymbol{k} \cdot \mathcal{A}(\boldsymbol{k})-\int_{\mathrm{HBZ}} d^{2} k \mathcal{F}(\boldsymbol{k})\right](\bmod 2)$,

where HBZ denotes half the Brillouin zone. $\mathcal{A}(\boldsymbol{k})=$ $\sum_{n}\left\langle u_{n}(\boldsymbol{k}) \mid \nabla_{n} u_{n}(\boldsymbol{k})\right\rangle$ is the Berry gauge potential and the Berry field strength is written as $\mathcal{F}=\nabla_{\mathbf{k}} \times\left.\mathcal{A}(\boldsymbol{k})\right|_{z}$ where $u_{n}(\boldsymbol{k})$ is the periodic part of the Bloch state with band index $n$ and the summation runs over all occupied states. According to Stokes' theorem, it is obvious that if $\mathcal{A}$ and $\mathcal{F}$ have the same gauge which is smooth over HBZ, the result will vanish. Therefore, one needs to fix the gauge with some additional constraints [42]. By choosing a gauge in which the corresponding states fulfill the TRS constraints in addition to the periodicity of the $k$ points, which are related by a reciprocal lattice $\boldsymbol{G}$, the gauge fixing procedure is complete and the returned results of $\mathbb{Z}_{2}=0$ or $\mathbb{Z}_{2}=1$ represent the trivial and topological phases, respectively. In the case of phosphorene, where bands cross or degeneracies are present in the energy spectrum, the Berry potential and Berry field strength must be extended to non-Abelian gauge field analogies [43] associated with a ground-state multiplet $|\psi(k)\rangle=\left(\left|u_{1}(k)\right\rangle, \ldots,\left|u_{2 M}(k)\right\rangle\right)$ in the equation $\mathcal{H}(k)\left|u_{n}(k)\right\rangle=E_{n}(k)\left|u_{n}(k)\right\rangle$.

Based on the above extension, the discretized Brillouin zone version [44] of Eq. (18) for numerical computing the $\mathbb{Z}_{2}$ invariant is written as

$$
\mathbb{Z}_{2}=\frac{1}{2 \pi i}\left[\sum_{k_{l} \in \partial \mathrm{HBZ}} A_{x}\left(k_{l}\right)-\sum_{k_{l} \in \mathrm{HBZ}} F_{x y}\left(k_{l}\right)\right](\bmod 2),
$$

where each site in the square lattice of the Brillouin zone of phosphorene is labeled by $k_{l}$ and $l$ specifies a plaquette with so-called unimodular link variable

$$
U_{\mu}\left(k_{l}\right)=\frac{\operatorname{det} \psi^{\dagger}\left(k_{l}\right) \psi\left(k_{l}+\hat{\mu}\right)}{\left|\operatorname{det} \psi^{\dagger}\left(k_{l}\right) \psi\left(k_{l}+\hat{\mu}\right)\right|},
$$

where $\hat{\mu}$ denotes a unit vector in $x-y$ plane. Such a link variable allows us to define the Berry potential and Berry field as

$$
\begin{gathered}
A_{x}\left(k_{l}\right)=\ln U_{x}\left(k_{l}\right), \\
F_{x y}\left(k_{l}\right)=\ln \frac{U_{x}\left(k_{l}\right) U_{y}\left(k_{l}+\hat{x}\right)}{U_{y}\left(k_{l}\right) U_{x}\left(k_{l}+\hat{y}\right)} .
\end{gathered}
$$

Berry potential and Berry field strength are both defined within the branch of $A_{x}\left(k_{l}\right) / i \in(-\pi, \pi)$ and $F_{x y}\left(k_{l}\right) / i \in(-\pi, \pi)$.

Figure 4 shows the results of $\mathbb{Z}_{2}$ corresponding to the energy bands in Fig. 2. As can be seen, at the critical strain of $11.8 \%$, which is consistent with the condition of $\varepsilon_{z}>E_{g}^{0} / \eta_{z}$ for band inversion, the $\mathbb{Z}_{2}$ invariant jumps from 0 to 1 . This demonstrates a topological phase transition in the electronic properties of phosphorene. According to Eq. (17), another way to observe a topological phase transition in phosphorene is by applying in-plane compressive biaxial strain at a fixed value of tensile strain in the $z$ direction. Figure 5 shows the numerically computed $\mathbb{Z}_{2}$ phase diagrams as a function of $\varepsilon_{x}$ and $\varepsilon_{y}$ at a

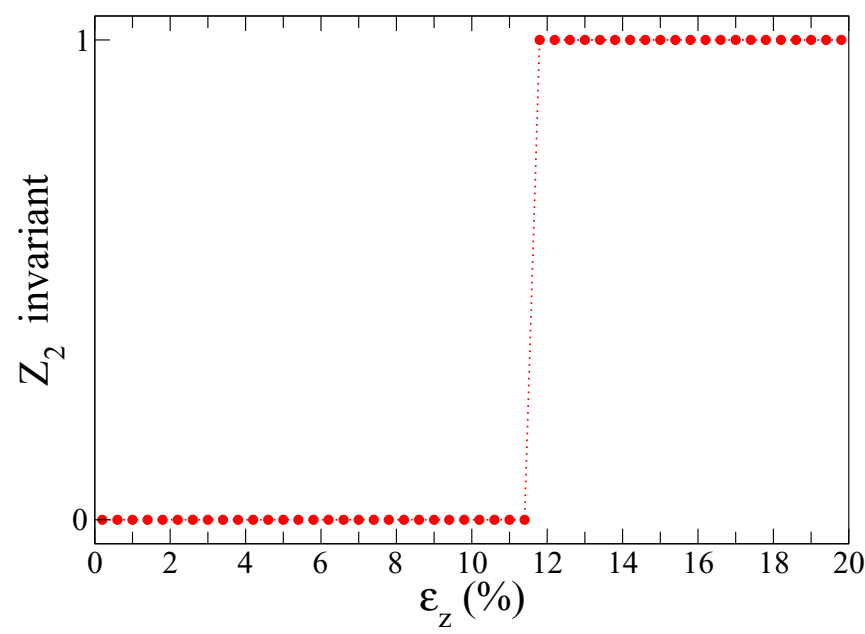

FIG. 4. Calculation of $\mathbb{Z}_{2}$ invariant of phosphorene in the presence of perpendicular tensile strain. The critical value for the topological phase transition is $11.8 \%$. 

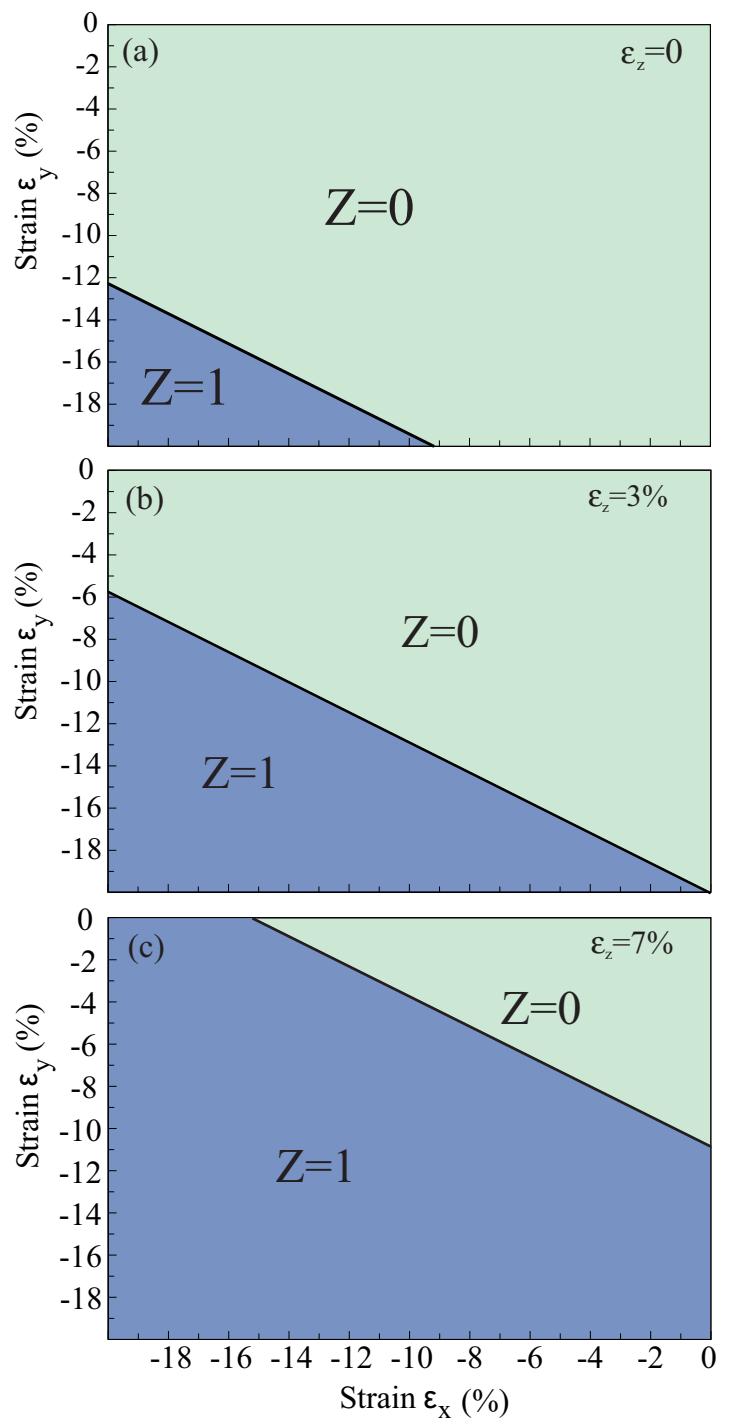

FIG. 5. Phase diagrams of the $\mathbb{Z}_{2}$ invariant as function of $\varepsilon_{x}$ and $\varepsilon_{y}$ for different values of $\varepsilon_{z}^{c}$. The linear boundaries distinguish the two topologically different phases according to the gap-closing condition of $\eta_{x} \varepsilon_{x}+\eta_{y} \varepsilon_{y}=E_{g}^{0}-\eta_{z} \varepsilon_{z}^{c}$.

fixed value of $\varepsilon_{z}$. As can be seen, there is a linear border between the two distinct topological phases that corresponds to the regimes before and after the gap closing condition of $\eta_{x} \varepsilon_{x}+\eta_{y} \varepsilon_{y}=E_{g}^{0}-\eta_{z} \varepsilon_{z}^{c}$, where $\varepsilon_{z}^{c}$ is a fixed value of strain in the direction of $z$.

It is worth mentioning that the relatively large bulk band gap of monolayer phosphorene necessitates a rather large value of strain in order to observe band inversion. As mentioned before, according to DFT calculations, this is accompanied by an upward shift of a new valance band maximum (VBM). After a critical percentage of strain, a direct band touching occurs, which is characterized by a TI phase. However, further increase of strain leads to a metal phase and because the topological nature does not change, the system may fall into the TM phase. Our model cannot predict the VBM upward shift; hence, in spite of demonstrating the change of the topological phase, it cannot distinguish between the TI and TM phases.
Note that our approach can be simply extended to the case of few-layer phosphorene in which we expect to observe the topological phase transition at lower strain values, due to the fact that the interlayer hoppings result in a smaller gap [45].

\section{B. Electronic properties of phosphorene nanoribbons under strain}

In this subsection, we investigate the evolution of the band structure of phosphorene nanoribbons in the presence of inplane and perpendicular strain. In the following, we refer to the width of zPNRs as $N_{z}$-zPNR with $N_{z}$ being the number of zigzag chains across the ribbon width. As we showed in the previous section, a topological phase transition occurs in the band spectrum of phosphorene. This should lead to the formation of topologically protected edge states in the band structure of the corresponding nanoribbons. We obtain the eigenvalues and eigenvectors using the matrix

$$
M_{i \alpha, j \beta}(\mathbf{k})=\sum_{m n} \tau_{m i \alpha, n j \beta} e^{i \mathbf{k} \cdot \mathbf{R}_{m n}},
$$

where $e^{i \mathbf{k} \cdot \mathbf{R}_{m n}}$ are the 1D Bloch wave functions. $m, n$ denote supercells; $i, j$ are the basis sites in a supercell, and $\alpha, \beta$ denote the spin degree of freedom. $\mathbf{k}$ is the wave vector, and $\mathbf{R}_{m n}$ represents a Bravais lattice vector. $\tau_{m i \alpha, n j \beta}$ are the hopping integrals with usual SOC or intrinsic Rashba coupling that are conveniently defined between the basis site $i$ with spin $\alpha$ of supercell $m$ and the basis site $j$ with spin $\beta$ of unit cell $n$.

Note that Eq. (23) is related to the energy spectrum of nanoribbons that are not edge passivated. The experimental realization of such nanoribbons with pristine edges in lowdimensional materials as graphene is well known [46] and may be extended to the case of phosphorene nanoribbons. However, the stability of such ribbons is important from the experimental point of view. Formation energy studies [47] showed that pristine phosphorene nanoribbons are stable especially for ribbon widths which we have considered in this paper.

The emergence of quasiflat bands which are detached completely from the bulk bands due to the special structure of phosphorene are well known $[27,39,48]$. As shown in Fig. 6(a), there are topologically nonprotected edge modes in the $1 \mathrm{D}$ bands of a typical zPNR (the results are for $N_{z}=100$ ). These quasiflat bands have been used to propose a field-effect transistor driven by an in-plane electric field [27,39]. However, since pristine bulk phosphorene is a trivial insulator, the existence of topologically nonprotected edge modes in the corresponding nanoribbons which can be affected by environmental conditions such as disorder or impurities may not be a good candidate for practical use. As an example, we consider the zigzag nanoribbon in the presence of perpendicular strain. The behavior in the presence of other types of strain is similar to this case. As can be seen in Figs. 6(b) and 6(c), by increasing strain the bulk gap of the nanoribbon gradually decreases and after a critical strain, where a band inversion occurs in the bulk spectrum, the corresponding edge states in the ribbon cross the gap which demonstrates a topological insulator phase. Owing to the dependence of the nanoribbon gap on the ribbon width, the critical strain for driving it to a topological insulator phase depends on the width as well. If we consider ribbons with 


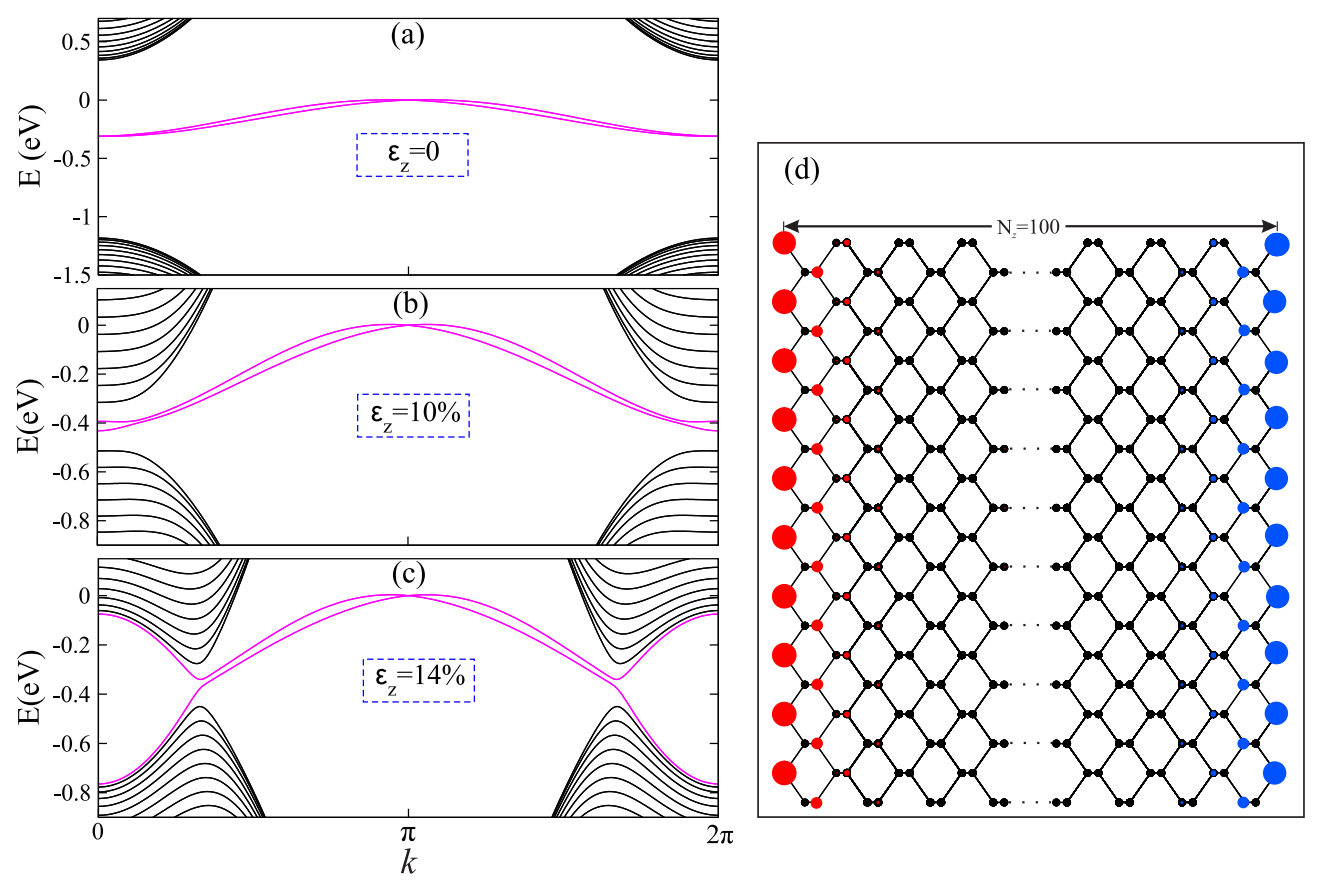

FIG. 6. 1 D energy bands for a typical phosphorene nanoribbon with $N_{z}=100(\sim 23 \mathrm{~nm})$ in case (a) without strain, (b) $\varepsilon_{z}=10 \%$, and (c) $\varepsilon_{z}=14 \%$. (d) The amplitude probability of the topological edge modes living on opposite edges for a definite $k$ point.

very large widths, the critical value approaches the critical strain value of bulk $11.8 \%$ that we have calculated in previous section.

The anisotropic structure of phosphorene results in a large bulk gap for zigzag nanoribbons with experimentally accessible widths. This makes strained zPNRs ideal systems for observing topological states even at room temperature. As shown in Fig. 6(c) for a zigzag nanoribbon of width $\sim 23 \mathrm{~nm}$ this gap is about $200 \mathrm{meV}$ which is much larger than room-temperature thermal energy. We have calculated numerically these bulk gaps for relatively large ribbons up to a width of $100 \mathrm{~nm}$ and found that the mentioned gaps are at least three orders of magnitude larger than the thermal energy at room temperature. It is worth mentioning that such a typical ribbon width is wide enough to prevent from overlapping of edge states living on opposite sides of the ribbon. The corresponding amplitude probability of the topological edge modes of Fig. 6(c) which have amplitude on opposite edges are shown in Fig. 6(d) for a definite $k$ point. The amplitude of the wave functions drop very quickly along the width of the ribbon demonstrating that the nanoribbon width is wide enough to prevent quantum tunneling. Such excellent properties can pave the way for utilizing it in device applications.

\section{CONCLUSIONS}

In summary, we derived a spin-orbit model Hamiltonian based on the structural and electronic properties of phosphorene that captures the main physical properties of spin-orbit related subjects. Then we showed in the frame of this TB model that gap engineering of phosphorene by axial strains can lead to a topological phase transition in the electronic properties of phosphorene. In spite of the relatively small gap induced by SOC in bulk monolayer phosphorene, we predict that due to the special puckered structure of phosphorene, zigzag nanoribbons in the regime of TI have topologically protected edge states with rather large bulk band gaps of about $200 \mathrm{meV}$ for a typical ribbon of width $\sim 23 \mathrm{~nm}$. Such gaps are larger that the thermal energy at room temperature and are therefore sufficiently large for practical device engineering at room temperature.

\section{ACKNOWLEDGMENTS}

This work was supported by Ministry of Science, Research and Technology, Iran. M.Z. acknowledges support as a postdoctoral fellow of the Flemish Research Foundation (FWO-Vl).
[1] C. L. Kane and E. J. Mele, Phys. Rev. Lett. 95, 226801 (2005).

[2] M. Z. Hasan and C. L. Kane, Rev. Mod. Phys. 82, 3045 (2010).

[3] J. E. Moore, Nature (London) 464, 194 (2010).

[4] X.-L. Qi and S.-C. Zhang, Rev. Mod. Phys. 83, 1057 (2011).

[5] L. Fu, C. L. Kane, and E. J. Mele, Phys. Rev. Lett. 98, 106803 (2007).

[6] B. A. Bernevig, T. L. Hughes, and S.-C. Zhang, Science 314, 1757 (2006).
[7] Y. Ren, Z. Qiao, and Q. Niu, Rep. Prog. Phys. 79, 066501 (2016).

[8] Y. Ma, Y. Dai, W. Wei, B. Huang, and M.-H. Whangbo, Sci. Rep. 4, 7297 (2014).

[9] M. Ezawa, Phys. Rev. Lett. 109, 055502 (2012).

[10] S. P. Koenig, R. A. Doganov, H. Schmidt, A. Castro Neto, and B. Oezyilmaz, Appl. Phys. Lett. 104, 103106 (2014).

[11] L. Li, Y. Yu, G. J. Ye, Q. Ge, X. Ou, H. Wu, D. Feng, X. H. Chen, and Y. Zhang, Nat. Nanotechnol. 9, 372 (2014). 
[12] F. Xia, H. Wang, and Y. Jia, Nat. Commun. 5, 4458 (2014).

[13] R. Fei, V. Tran, and L. Yang, Phys. Rev. B 91, 195319 (2015).

[14] J. Kim, S. S. Baik, S. H. Ryu, Y. Sohn, S. Park, B.-G. Park, J. Denlinger, Y. Yi, H. J. Choi, and K. S. Kim, Science 349, 723 (2015).

[15] Z. J. Xiang, G. J. Ye, C. Shang, B. Lei, N. Z. Wang, K. S. Yang, D. Liu, F. B. Meng, X. G. Luo, L. J. Zou, Z. Sun, Y. Zhang, and X. H. Chen, Phys. Rev. Lett. 115, 186403 (2015).

[16] Q. Liu, X. Zhang, L. Abdalla, A. Fazzio, and A. Zunger, Nano Lett. 15, 1222 (2015).

[17] T. Zhang, J.-H. Lin, Y.-M. Yu, X.-R. Chen, and W.-M. Liu, Sci. Rep. 5, 13927 (2015).

[18] Q. Wei and X. Peng, Appl. Phys. Lett. 104, 251915 (2014).

[19] X. Peng, Q. Wei, and A. Copple, Phys. Rev. B 90, 085402 (2014).

[20] A. N. Rudenko and M. I. Katsnelson, Phys. Rev. B 89, 201408 (2014).

[21] L. Liang, J. Wang, W. Lin, B. G. Sumpter, V. Meunier, and M. Pan, Nano Lett. 14, 6400 (2014).

[22] V. Tran, R. Soklaski, Y. Liang, and L. Yang, Phys. Rev. B 89, 235319 (2014).

[23] J. Qiao, X. Kong, Z.-X. Hu, F. Yang, and W. Ji, Nat. Commun. 5, 4475 (2014).

[24] Y. Takao and A. Morita, Physica B+C 105, 93 (1981).

[25] R. Fei and L. Yang, Nano Lett. 14, 2884 (2014).

[26] Y. Li, S. Yang, and J. Li, J. Phys. Chem. C 118, 23970 (2014).

[27] E. Taghizadeh Sisakht, M. H. Zare, and F. Fazileh, Phys. Rev. B 91, 085409 (2015).

[28] C.-C. Liu, H. Jiang, and Y. Yao, Phys. Rev. B 84, 195430 (2011).

[29] A. Castellanos-Gomez, L. Vicarelli, E. Prada, J. O. Island, K. Narasimha-Acharya, S. I. Blanter, D. J. Groenendijk, M.
Buscema, G. A. Steele, J. Alvarez, H. W. Zandbergen, J. J. Palacios, and H. S. J. van der Zant, 2D Mater. 1, 025001 (2014).

[30] A. S. Rodin, A. Carvalho, and A. H. Castro Neto, Phys. Rev. Lett. 112, 176801 (2014).

[31] C. Wang, Q. Xia, Y. Nie, and G. Guo, Appl. Phys. Lett. 117, 124302 (2015).

[32] G. Q. Huang and Z. W. Xing, arXiv:1409.7284.

[33] J.-W. Jiang and H. S. Park, Phys. Rev. B 91, 235118 (2015).

[34] Y. Mohammadi and B. A. Nia, Superlatt. Microstruct. 89, 204 (2016).

[35] H. Duan, M. Yang, and R. Wang, Physica E 81, 177 (2016).

[36] B. Sa, Y.-L. Li, J. Qi, R. Ahuja, and Z. Sun, J. Phys. Chem. C 118, 26560 (2014).

[37] W. A. Harrison, Elementary Electronic Structure (World Scientific, Singapore, 1999).

[38] H. Tang, J.-W. Jiang, B.-S. Wang, and Z.-B. Su, Solid State Commun. 149, 82 (2009).

[39] M. Ezawa, New J. Phys. 16, 115004 (2014).

[40] A. A. Soluyanov, Ph.D. thesis, Topological aspects of band theory, Rutgers University, 2012.

[41] L. Fu and C. L. Kane, Phys. Rev. B 74, 195312 (2006).

[42] A. A. Soluyanov and D. Vanderbilt, Phys. Rev. B 83, 035108 (2011).

[43] T. Fukui, Y. Hatsugai, and H. Suzuki, J. Phys. Soc. Jpn. 74, 1674 (2005).

[44] T. Fukui and Y. Hatsugai, J. Phys. Soc. Jpn. 76, 053702 (2007).

[45] Y. Cai, G. Zhang, and Y.-W. Zhang, Sci. Rep. 4, 6677 (2014).

[46] X. Zhang, J. Xin, and F. Ding, Nanoscale 5, 2556 (2013).

[47] A. Carvalho, A. S. Rodin, and A. H. Castro Neto, Europhys. Lett. 108, 47005 (2014).

[48] M. M. Grujić, M. Ezawa, M. Ž. Tadić, and F. M. Peeters, Phys. Rev. B 93, 245413 (2016). 